\title{
POSITIVELY CURVED DEFORMATIONS OF INVARIANT RIEMANNIAN METRICS
}

\begin{abstract}
ALAN WEINSTEIN ${ }^{1}$
ABstracr. Let $K_{\gamma}$ denote the sectional curvature function of the Riemannian metric $\gamma$ on a manifold $M$. Suppose $M$ admits no metric $\gamma$ invariant under the action of a compact group $G$ and having $K_{\gamma}>0$. It is shown that a $G$-invariant metric $\gamma(0)$ with $K_{\gamma(0)} \geqq 0$ cannot be embedded in a 1-parameter family $\gamma(t)$ for which $\left[d K_{\gamma(t)} / d t\right]_{t=0}$ is positive wherever $K_{\gamma(0)}$ is zero.
\end{abstract}

The sectional curvature of the Riemannian metric $\gamma$ on the manifold $M$ is a function $K_{\gamma}$ on the bundle $P(M)$ of tangent 2-planes to $M$. Denote by $Z_{\gamma} \subseteq P(M)$ the set of zeros of $K_{\gamma}$. A smooth 1-parameter family $\gamma(t)$ will be called positive if $K_{\gamma(0)}$ is nonnegative and if $\left[d K_{\gamma(t)} / d t\right]_{t=0}$ is positive on $Z_{\gamma(0)}$. If $M$ is compact, $K_{\gamma(\epsilon)}$ is positive for small positive $\epsilon$. Berger [2] showed that if $M$ is compact, a family on $M$ for which $\gamma(0)$ is a product metric cannot be positive. The following theorem, combined with the results cited after its proof, gives other examples of metrics which cannot be embedded in positive families.

THEOREM. If $M$ is a compact manifold which admits no metric of positive sectional curvalure invariant under a given compact group $G$ of diffeomorphisms, then a family $\gamma(t)$ of metrics on $M$ for which $\gamma(0)$ is G-invariant cannot be positive.

Proof. We prove the contrapositive. Suppose that $\gamma(t)$ is $G$-invariant, and define

$$
\delta(t)=\int_{G} g^{*} \gamma(t) d g,
$$

where $d g$ is the Haar measure on $G$ normalized so that $G$ has total measure $1 . \delta(0)=\gamma(0)$, and $\delta(t)$ is $G$-invariant for each $t$. Denoting by $K_{*}$ the differential at $\delta(0)=\gamma(0)$ of the mapping $\gamma \mapsto K_{\gamma}$, we have

Received by the editors January 5, 1970.

AMS 1969 subject classifications. Primary 5372, 5366.

Key words and phrases. Invariant Riemannian metric, family of Riemannian metrics, positive sectional curvature, homogeneous space, Haar measure.

1 Research partially supported by NSF grant GP-13348. 


$$
\begin{aligned}
]_{\delta(t)} d K_{\delta(t)}\right]_{t=0} & =K_{*}[d \delta(t) / d t]_{t=0} \\
& =K_{*} \int_{G} g^{*}[d \gamma(t) / d t]_{t=0} d g \\
& =\int_{G} K_{*} g^{*}[d \gamma(t) / d t]_{t=0} d g \\
& =\int_{G} g^{*} K_{*}[d \gamma(t) / d t]_{t=0} d g \\
& =\int_{G} g^{*}\left[d K_{\gamma(t)} / d t\right]_{t=0} d g .
\end{aligned}
$$

The second and third lines are equal because $K_{*}$ is a linear partial differential operator. The $g^{*}$ in the last two lines refers to the induced action of $G$ on $P(M)$. The relation $K_{*} g^{*}=g^{*} K_{*}$, valid for any diffeomorphism $g$, expresses the naturality of the operation $\gamma \mapsto K_{\gamma}$.

Since $Z_{\gamma(0)}$ is invariant under this action, the integrand in the last integral is positive on $Z_{\gamma(0)}$ for each $g \in G$. It follows that $\left[d K_{\delta(t)} / d t\right]_{t=0}$ is positive on $Z_{\gamma(0)}$, and $K_{\delta(\epsilon)}$ is positive for small positive $\epsilon$. Since $\delta(\epsilon)$ is $G$-invariant, the contrapositive theorem is proven.

The interest of this result rests on the fact that many compact homogeneous spaces $G / H$ are known to admit no $G$-invariant metric of positive curvature. If the linear isotropy group of $G$ acting on $G / H$ is irreducible, then any $G$-invariant metric is proportional to the normal one, and Berger [1] has shown that every homogeneous space with normal metric of positive curvature is diffeomorphic to a symmetric space of rank one or to one of two exceptional spaces. Recently, Wallach [3] has shown that if $G / H$ is even-dimensional and admits a $G$-invariant metric of positive curvature then $M$ is diffeomorphic to a symmetric space of rank one or to one of two other exceptional spaces. It is unknown whether Wallach's exceptional spaces actually admit homogeneous metrics of positive curvature.

\section{REFERENCES}

1. M. Berger, Les variêtés riemanniennes homogènes normales simplement connexes d courbure strictement positive, Ann. Scuola Norm. Sup. Pisa (3) 15 (1961), 179-246. MR 24 \#A2919.

2. - Trois remarques sur les variêtés riemannienes à courbure positive, $\mathrm{C} . \mathrm{R}$. Acad. Sci. Paris Sér. A-B 263 (1966), A76-A78. MR 33 \#7966.

3. N. Wallach, Homogeneous positively pinched riemannian manifolds, (to appear).

University of California, Berkeley, California 94720 